\title{
Evaluación de la calidad en la prestación de servicios de salud por medio de series de tiempo enmarcado en la metodología Seis Sigma
}

\author{
Evaluation of the quality of health care provision using time series analysis based on Six Sigma \\ Avaliação da qualidade da prestação de cuidados de saúde utilizando análise de séries temporais \\ baseada no Seis Sigma \\ Miguel Oswaldo Pérez Pulido, ${ }^{\mathrm{a} *}$ Giampaolo Orlandoni Merli, Josefa Ramoni Perazzi, Miguel Valbuena Vence. \\ ${ }^{* a}$ Magíster en Estadística, Universidad de Santander, Bucaramanga, Santander. (1) \\ ${ }^{b}$ Doctor en Estadística, Universidad de Santander, Bucaramanga, Santander.(1) \\ ${ }^{\circ}$ Doctor en Economía, Universidad de Santander, Bucaramanga, Santander.(1) \\ ${ }^{` M a g i s t e r ~ e n ~ G e s t i o ́ n ~ P u ́ b l i c a ~ y ~ G o b i e r n o, ~ U N I P S S A M ~ I P S ~-~ C l i ́ n i c a ~ d e ~ s a l u ́ d ~ m e n t a l ~ d e ~ A g u a c h i c a ~(C e s a r) .(1) ~}$
}

\begin{abstract}
Forma de citar: Pérez, M. Orlandoni, G. Ramoni, J. Valbuena, M. (2016). Evaluación de la calidad en la prestación de servicios de salud por medio de series de tiempo enmarcado en la metodología seis sigma. Encuentro Internacional en Educación Matemática ISSN 2539-1885. La Educación Matemática como Herramienta en el Desempeño Profesional Docente.Cúcuta, Colombia. 73 - 77.
\end{abstract}

\begin{abstract}
Antecedentes: Dada su importancia, la salud en Colombia es un tema fundamental en los programas de gobierno tanto a nivel nacional, como regional y local. Garantizar el acceso a servicios de salud de calidades una de las condiciones necesarias para la reducción de la pobreza y de la desigualdad en el país. Conscientes de esta necesidad, las autoridades del ESE Hospital Lázaro Hernández (HLH),(San Alberto, Departamento del Cesar, Colombia) se proponen elevar la calidad de los servicios que dicho ente proporciona a la comunidad, la cual se caracteriza por tener altos niveles de pobreza, además de una alta incidencia de enfermedades tropicales y elevado porcentaje de población flotante procedente de tres diferentes departamentos (Cesar, Norte de Santander, Santander), debido a su ubicación geográfica fronteriza. Objetivo: Contribuir a mejorar la calidad de los servicios de salud de dicho centro asistencial a través de la aplicación de la metodología Seis Sigma. Métodos: Estudio transversal sobre percepción de la calidad en servicios de salud, donde se presentan los avances preliminares de la investigación, abordando temas de calidad en salud, como el comportamiento de los tiempos de espera y el número de pacientes atendidos en el área de urgencias durante el año 2015; los resultados de la medición de percepción de calidad por los usuarios del Hospital frente a la atención en las áreas de urgencias y consulta externa, recabados mediante la encuesta SERVQUAL, instrumento que mide la percepción de calidad en servicios de salud. La población objetivo está conformada por los pacientes atendidos durante los meses de marzo y abril de 2015 en las áreas de urgencias y consulta externa del HLHL. Los resultados obtenidos se basan en el análisis de las series de tiempos de espera y número de pacientes atendidos, para evaluar la percepción de calidad en atención, usando la herramienta computacional Statgraphics XVI. Resultados: La calificación global de percepción, permite concluir que alrededor del $50 \%$ de los pacientes tienen percepciones bajas en las dimensiones evaluadas de calidad de los servicios prestados por el HLH. Conclusiones: Los resultados indican que los pacientes están inconformes con la calidad del servicio que presta la ESE HLH, la cual es percibida como baja. Ello permite recomendar a las directivas del hospital revisar los procesos relacionados con la calidad de los servicios prestados, y proponer políticas de mejoramiento de acuerdo a las necesidades y las condiciones de los usuarios.
\end{abstract}

Palabras clave Análisis factorial confirmatorio, calidad en salud, indicadores de gestión en salud, seis sigma, series de tiempo, SERVQUAL.

Background: Given its obvious importance, health in Colombia is a fundamental issue in government programs at national, regional and local levels. Ensuring access to quality health services is pivotal to reducing poverty and inequality in the country. Cognizant of this need, authorities of the ESE Hospital Lázaro Hernández (HLH), (San Alberto, Department of Cesar, Colombia) intend to augment the quality of community services provided by the hospital. Said community is characterized by soaring levels of poverty, a high incidence of tropical diseases and an elevated floating population percentage coming from three different departments (Cesar, Norte de Santander, Santander), due to their geographic border location. Objective: To contribute to improving quality of health services of said assistance center through the application of the Six Sigma methodology. Methods: A cross-sectional study on the perception of quality in health services, presenting the preliminary research advances, addressing health quality issues such as waiting-time performance and number of patients attended in the emergency area during 2015. The results of the measurement of service quality perception related to attention in areas such as emergency and external consultation, were collected through the SERVQUAL survey, an instrument that measures perception of quality in health services. The target population consists of patients treated during March and April of 2015 in the emergency and outpatient areas of the HLH. Results obtained are based on analysis of the waiting-time series and number of patients attended, in order to evaluate the perception of quality of services, using Statgraphics XVI, a computational tool. Results: The overall perception rating indicates that about $50 \%$ of the patients have low perceptions on the quality dimensions of services provided by HLH. Conclusions: The results indicate that patients are not satisfied with the perceived low quality services provided by the ESE HLH. It can therefore be recommended that hospital directives review processes related to the quality of services provided, as well as propose improvement policies according to the needs and conditions of the users.

Keywords Confirmatory factorial analysis, health quality, health management indicators, six sigma, time series, SERVQUAL.

* Autor para correspondencia miguel.perez@udes.edu.co

2590-9215@ 2017 Universidad Francisco de Paula Santander. Este es un artículo bajo la licencia CCBY 
Antecedentes: Dada a sua importância, a saúde na Colômbia é uma questão fundamental nos programas governamentais a nível nacional, regional e local. Garantir o acesso a serviços de saúde de qualidade é uma das condições necessárias para reduzir a pobreza e a desigualdade no país. Conscientes dessa necessidade, as autoridades do Hospital ESZ Lázaro Hernández (HLH) (San Alberto, Departamento de Cesar, Colômbia) pretendem elevar a qualidade dos serviços prestados pelo hospital à comunidade, que se caracteriza por altos níveis de pobreza, além de uma alta incidência de doenças tropicais e alta porcentagem de população flutuante proveniente de três diferentes departamentos (Cesar, Norte de Santander, Santander), devido à sua localização geográfica. Objetivo: Contribuir para melhorar a qualidade dos serviços de saúde deste centro de atendimento por meio da aplicação da metodologia Seis Sigma. Métodos: Estudo transversal sobre a percepção da qualidade em serviços de saúde, apresentando os avanços preliminares da pesquisa, abordando questões de qualidade em saúde, como desempenho no tempo de espera e número de pacientes atendidos na área de emergência durante o ano de 2015; os resultados da mensuração da percepção da qualidade do serviço relacionados à atenção em áreas como consulta externa e de emergência, coletados por meio da pesquisa SERVQUAL, instrumento que mede a percepção de qualidade nos serviços de saúde. A população alvo é composta por pacientes tratados durante os meses de março e abril de 2015 nas áreas de emergência e ambulatoriais do HLH. Os resultados obtidos baseiam-se na análise das séries temporais dos pacientes e no número de pacientes atendidos, para avaliar a percepção da qualidade dos serviços, utilizando a ferramenta computacional Statgraphics XVI. Resultados: O índice global de percepção indica que cerca de 50\% dos pacientes têm baixa percepção sobre as dimensões de qualidade dos serviços prestados pela HLH.

Conclusões: Os resultados indicam que os pacientes não estão satisfeitos com a qualidade dos serviços prestados pela ESE HLH, que é percebida como muito baixa. Isso nos permite recomendar diretrizes hospitalares para revisar os processos relacionados à qualidade dos serviços prestados e propor políticas de melhoria de acordo com as necessidades e condições dos usuários.

Palavras chave Análise fatorial confirmatória, qualidade da saúde, indicadores de gestão em saúde, seis sigma, série temporal, SERVQUAL.

\section{Introducción}

La mejora en la calidad de los servicios de salud es una necesidad muy sentida en Colombia. No obstante, los avances que se han logrado en materia de reformas en pro de elevar la calidad de estos servicios, se reconoce que aún falta mucho camino por recorrer. Prueba de ello es el hecho de que la mejora en los servicios de salud forma parte fundamental de planes de gobierno a nivel nacional y regional: el Plan de desarrollo del Cesar "Prosperidad a salvo" incluye dentro del programa "de frente contra la pobreza" mejorar los servicios de salud en términos de calidad, eficiencia, equidad y sostenibilidad. Si bien el proyecto se está llevando a cabo en el Cesar, se propone generar estrategias que permitan su replicabilidad en instalaciones sanitarias de Santander y en otros departamentos.

Así, el Plan de Desarrollo de Bucaramanga 2010-2015 planteaba entre sus dimensiones la de la Sostenibilidad Social y Económica, de la cual forma parte el eje programático Salud y Protección Social. El Plan Nacional de Colombia (2014-2018) propone entre sus objetivos para una Colombia equitativa y sin pobreza, la provisión de servicios de salud de calidad (DNP, 2014). El Proyecto Diamante del Caribe y Santanderes (FINDETER et al, 2014), que involucra tanto al Cesar como a Santander, establece que para que la alianza fructifique y la calidad de vida de las ciudades de los Departamentos mejore, debe fortalecerse, entre otros, el sector salud.

En este trabajo se presentan los avances preliminares de la investigación, abordando temas de calidad en salud, como el comportamiento de los tiempos de espera de pacientes atendidos en el área de urgencias durante el año 2016 en el ESE Hospital Lázaro Hernández Lara (HLH), del municipio de San Alberto, Cesar; los resultados de la medición de percepción de calidad por los usuarios del Hospital frente a la atención en las áreas de urgencias y consulta externa. Para esto, a través de la metodología Seis Sigma, se tomará como estrategia de mejora continua de los procesos que se enfoca en localizar y reducir la variabilidad de los procesos, productos/servicios y los errores, defectos y retrasos asociados hasta alcanzar un nivel mínimo de excelencia. El trabajo computacional se hace mediante la herramienta computacional el Statgraphics XVI.

\section{Métodos}

Según Gutiérrez y De la Vara (2009), definen a Seis Sigma como "una estrategia que busca mejorar el desempeño de los procesos de una organización y reducir su variación; con ello, es posible encontrar y eliminar causas de los errores, defectos y retrasos en los procesos del negocio". Como lo señala Orlandoni (2012), la metodología Seis Sigma puede aplicarse para reducir tasa de defectos de productos, servicios y procesos existentes (DMAMC) y para la fase de diseño del producto y del proceso (DMADV).

La primera implica definir el problema o describir el proceso; definir y evaluar los sistemas de medición, garantizando respetabilidad y reproducibilidad; analizar las variables significativas del proceso; evaluar la estabilidad y capacidad del proceso, a través de graficas de control y finalmente, mejorar y optimizar el proceso y aplicar mecanismos de monitoreo.

Por su parte, DMADV se aplica a productos nuevos o proceso en prueba, para lo que se recurre al diseño estadístico, siguiendo las siguientes etapas: definición, medición, análisis, diseño y verificación. Sin embargo, según Ramírez et al. (2007) para que Seis Sigma funcione, se requiere que su filosofía y principios sean aplicados por todo el personal involucrado de arriba hacia abajo, para lo cual es menester el compromiso de la dirección superior, quienes deben estar realmente involucrados con la cultura organizacional de Seis Sigma. 
En la Figura 1, se muestran las etapas con actividades requeridas para cada una de ellas. Para la aplicación de esta metodología de mejoramiento, se deben seleccionar proyectos que tengan las siguientes características básicas:

1. Ser un proceso específico que contribuya a brindar un servicio superior.

2. Ser factible de realizar.

3. Tener un impacto medible en la mejora de la calidad del proceso.

4. Incrementar los niveles de productividad del proceso.

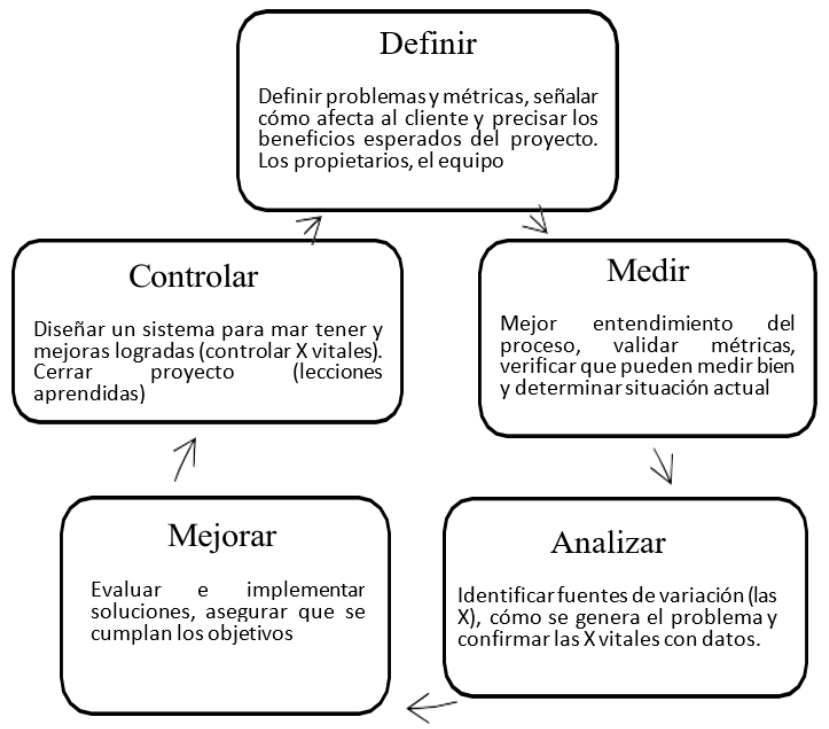

Figura 1. Las cinco etapas en la realización de un proyecto $60^{\circ}$ Fuente: Gutiérrez y De La Vara, Pág. 425.

En el presente estudio, se verifican las dimensiones medidas de percepción de calidad de acuerdo al instrumento SERVQUAL, que es un cuestionario con preguntas estandarizadas para la medición de la calidad del servicio; fue desarrollado por Valerie A. Zeithaml, A. Parasuraman y Leonard L. Berry, con el auspicio del Marketing Science Institute, y validado para América Latina por Michelsen Consulting, con el apoyo del nuevo Instituto Latinoamericano de Calidad en los Servicios.

Es uno de los instrumentos más citados en la literatura sobre calidad y el que mayor atención ha recibido por parte de autores de muy distintas disciplinas.

Además, hay que enfatizar que se trata de una medida de calidad percibida y, por tanto, no de la calidad técnica que ofrece el hospital. Diversas investigaciones acerca de la aplicación del modelo SERVQUAL concluyen que ha sido el más utilizado para la evaluación de la calidad de los servicios públicos.
En particular, Bigne et al. (1997), demuestran la fiabilidad de la escala SERVQUAL frente a otras metodologías para medir la calidad, fundamentalmente en tres servicios públicos: educación superior, transporte y salud. Este instrumento ha identificado cinco dimensiones de la calidad, de las cuales solo una es visible:

1.Tangibles: Como la parte visible de la oferta: la apariencia y limpieza de las instalaciones físicas, equipo, personal y materiales de comunicación.

2. Confiables: la habilidad para desarrollar el servicio prometido en forma acertada y como fue dicho.

3. Respuesta del personal: respuesta pronta y deseada, deseo de ayudar a los usuarios y de proveer el servicio con prontitud y esmero.

4. Seguridad: conocimiento y cortesía del personal y su habilidad para producir confianza.

5. Calidez o empatía: el cariño, la atención individualizadas que se provee a los usuarios, el deseo de comprender las necesidades precisas del cliente y encontrar la respuesta más adecuada.

Se utilizó la misma escala Likert que maneja SERVQUAL, reducida a 5 niveles, ya que ello permitió simplificar el llenado del cuestionario y la interpretación de la información que se obtuvo. El cuestionario fue adaptado de acuerdo a las condiciones sociales y culturales de los usuarios del ESE Hospital Lázaro Hernández Lara y solamente se midió lo que se percibía en la atención de los servicios de salud; no se midieron las expectativas o lo que se espera recibir, como lo indica la medición de la herramienta SERVQUAL.

\section{Resultados}

Análisis de los tiempos de espera de los pacientes en urgencias. Los tiempos de espera de pacientes atendidos en el área de urgencias durante el año 2015 en el ESE Hospital Lázaro Hernández Lara, se estudia mediante análisis de series de tiempo. De manera global, en promedio, un paciente espera aproximadamente 40 minutos para ser atendido por urgencias.

Los meses que registraron mayores tasas promedios de espera fueron febrero (50min) y agosto (57 min). El 40\% del total de pacientes atendidos, son de edades que oscilan entre 10-40 años, y el $90 \%$ de diagnósticos de consulta son por enfermedad general.

La Figura 2 muestra los tiempos de espera semanales promedio de los pacientes en el área de urgencias. 
Miguel Oswaldo Pérez Pulido, Giampaolo Orlandoni Merli, Josefa Ramoni Perazzi, Miguel Valbuena Vence

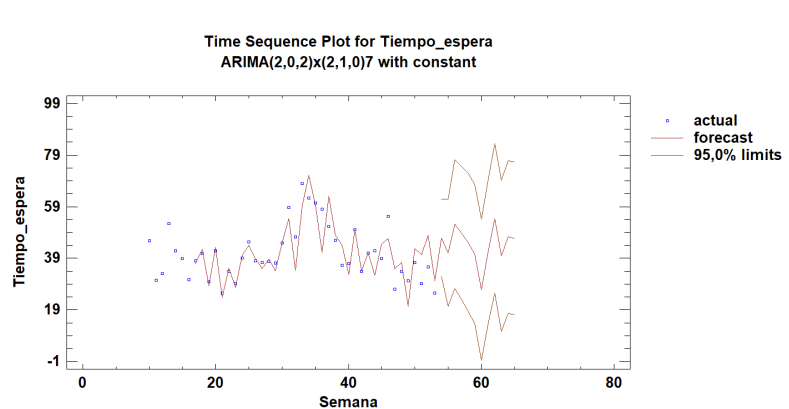

Figura 2. Time Sequence Plot for Promedio (Tiempo) ARIMA $(2,0,2) \mathrm{x}(2,1,0) 7$ Fuente: Datos suministrados por el Hospital Lázaro Hernández Lara 2016. Elaboración propia

El modelo que mejor ajusta a los datos es un ARIMA $(2,0,2) \times(2,1,0) 7$, con las siguientes características como lo muestra la Tabla I:

Tabla I. Estimación del modelo ARIMA para el Tiempo de Espera de pacientes en el Área de Urgencias.

\begin{tabular}{ccccc}
\hline Parámetro & Estimación & Error Est & $t$ & $p$-value \\
\hline$A R(1)$ & 1,805 & 0,033 & 54,2 & 0,000 \\
$A R(2)$ & $-0,990$ & 0,027 & & 0,000 \\
$M A(1)$ & 1,415 & 0,049 & 28,9 & 0,000 \\
$M A(2)$ & $-0,891$ & 0,064 & & 0,000 \\
$\operatorname{SAR}(1)$ & & 0,097 & $-3,2$ & 0,000 \\
$\operatorname{SAR}(2)$ & $-0,970$ & 0,042 & & 0,000 \\
\hline
\end{tabular}

Fuente: Backforecasting: yes, Estimated white noise variance $=22,99$ with 32 degrees of freedom Estimated white noise standard deviation $=4,79$

Este modelo genera predicciones aceptables. El diagnóstico del modelo de acuerdo a la Figura3, indica que los residuos son ruido blanco, basándose en el periodograma integrado y en el autocorrelograma de los residuos obtenidos en el análisis.

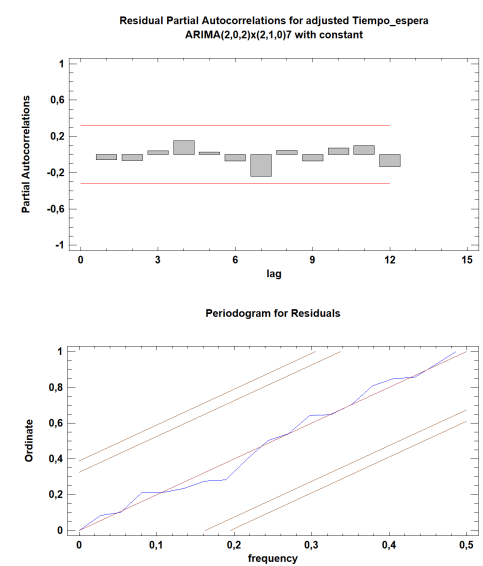

Figura 3. Diagnóstico del modelo ARIMA (Periodo grama y autocorrelo grama de residuos) de la Serie de tiempo de espera de pacientes en el área de urgencias. Fuente: Datos suministrados por el Hospital Lázaro Hernández Lara 2016. Elaboración propia
Análisis del promedio de pacientes atendidos por semana en urgencias. El pronóstico de suavización exponencial simple es óptimo para patrones de demanda aleatorios o nivelados donde se pretende eliminar el impacto de los elementos irregulares históricos mediante un enfoque en períodos de demanda reciente, este posee una ventaja sobre el modelo de promedio móvil ponderado ya que no requiere de una gran cantidad de períodos y de ponderaciones para lograr óptimos resultados. Para el análisis de la serie de tiempo "Numero de pacientes atendidos per cápita en el área de urgencias", se ajustó un modelo de suavización exponencial simple, por el hecho que la serie no presenta tendencia ni estacionalidad marcada. Este modelo se define como:

$$
Y_{t}^{\prime}=Y_{t-1}^{\prime}+\left(\propto *\left(Y_{t-1}^{\prime}-Y_{t-1}^{\prime}\right)\right)
$$

\section{Donde:}

$\boldsymbol{Y}_{t}^{\prime}$ : Promedio de pacientes atendidos en el periodo $t$.

$\boldsymbol{Y}_{t-1}^{\prime}:$ Pronóstico de pacientes atendidos en el período t-1.

$Y_{t}:$ Pacientes reales atendidos en el periodo $t-1$. $\propto$ : Coeficiente de Suavización (entre 0,0 y 1,0).

La Figura 4 muestra el modelo de suavización exponencial que mejor se ajustó al número de pacientes atendidos en el área de urgencias, con un coeficiente de suavización exponencial alfa de 0,36 y un RMSE de 23,65.

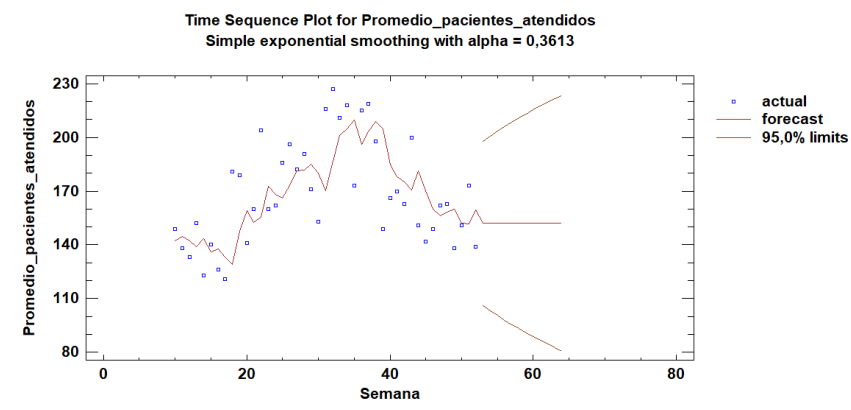

Figura 4. Serie de Tiempo con suavización exponencial simple del número promedio de pacientes atendidos en el área de urgencias. Fuente: Datos suministrados por el Hospital Lázaro Hernández Lara 2016. Elaboración propia

La Figura 5 muestra el diagnóstico del modelo de suavización exponencial simple del número de pacientes atendidos en el área de urgencias. Se observa que los residuos son ruido blanco, basándose en el periodograma integrado y en el autocorrelograma de los residuos obtenidos en el análisis.
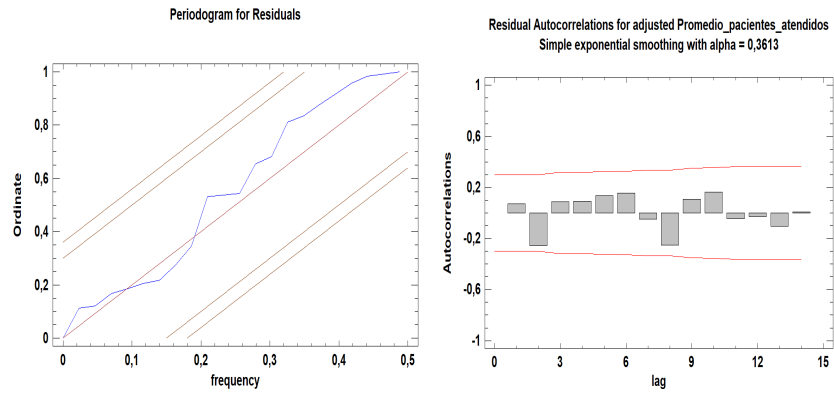

Figura 5. Diagnóstico del modelo de suavización exponencial simple (Periodo grama y autocorrelo grama de residuos) de la Serie de tiempo Número de pacientes atendidos en el área de urgencias.
Fuente: Datos suministrados por el Hospital l Lázaro Hernández Lara 2016 
Medición de la calidad de atención en el ESE Lázaro Hernández Lara Para tener una medición de la calidad prestada por el ESE Hospital Lázaro Hernández Lara, de acuerdo a la metodología SERVQUAL, se sumaron las puntuaciones de cada cuestionario para cada ítem de cada dimensión resultante del análisis factorial (ver Tabla II). Esta calificación se asignó de acuerdo a los cuartiles de las puntuaciones: "percepción baja" para puntuaciones menores al cuartil 2; "percepción media" entre el segundo y tercer cuartil y "percepción alta" para puntuaciones mayores al tercer cuartil.

\begin{tabular}{|c|c|c|c|c|c|c|c|c|c|c|}
\hline \multirow{2}{*}{ Dimensiones } & \multicolumn{2}{|c|}{$\begin{array}{l}\text { Empatía y } \\
\text { Seguridad }\end{array}$} & \multicolumn{2}{|c|}{$\begin{array}{l}\text { Capacidad } \\
\text { de Resp. }\end{array}$} & \multicolumn{2}{|c|}{ Fiabilidad } & \multicolumn{2}{|c|}{ Tangible } & \multicolumn{2}{|c|}{ Comodidad } \\
\hline & Obs & $\%$ & Obs & $\%$ & Obs & $\%$ & Obs & $\%$ & Obs & $\%$ \\
\hline Alta & 42 & 18.9 & 33 & 14.9 & 55 & 24.8 & 38 & 17.1 & 22 & 9.9 \\
\hline Media & 94 & 42.3 & 99 & 44.6 & 113 & 50.9 & 115 & 51.8 & 73 & 32.9 \\
\hline Baja & 86 & 38.7 & 90 & 40.5 & 54 & 24.3 & 69 & 31.1 & 127 & 57.2 \\
\hline
\end{tabular}

El $45 \%$ de los pacientes encuestados tiene percepciones bajas en capacidad de respuesta, el $40 \%$ percepción media y $15 \%$ percepción alta; el $42 \%$ tiene una percepción baja respecto a empatía y seguridad, mientras que un 56\% tiene una percepción media y alta; en materia de comodidad, el $33 \%$ tiene una percepción baja y un $67 \%$ percepciones medias y altas; en elementos tangibles, el 52\% tiene percepción baja y un $48 \%$ niveles medios y altos.

De acuerdo a lo anterior, el $51 \%$ de los pacientes tiene percepciones bajas en las dimensiones de calidad del servicio evaluadas, permitiendo recomendar a las directivas del hospital revisar los procesos relacionados a la calidad y proponer políticas de mejoramiento de acuerdo a las necesidades y las condiciones de los usuarios.

\section{Discusión}

Referente a la calidad de atención percibida en el ESE Hospital Lázaro Hernández Lara, de acuerdo a las dimensiones medidas de percepción de calidad de acuerdo al instrumento SERVQUAL, la dimensión "seguridad" aparece confundida con "empatía" y surge la dimensión "comodidad", separada de elementos "tangibles".

El $51 \%$ de los pacientes tiene una percepción baja en elementos tangibles; el $44 \%$ tiene percepción baja en capacidad de respuesta y un $32 \%$ en comodidad. La cuarta parte de los encuestados tiene una fiabilidad alta en los procesos de atención en salud del Hospital.
Para los tiempos de espera de los pacientes atendidos en el área de urgencias durante el año 2015, el modelo que mejor ajusta a los datos es un ARIMA $(2,0,2) \times(2,1,0) 7$ y una suavización exponencial para la serie del número de pacientes atendidos per cápita en el 2015, con un coeficiente de suavización exponencial alfa de 0,36 .

Un paciente espera aproximadamente 40 minutos para ser atendido en el área de urgencias; el 95\% de los diagnósticos en dicha área son por enfermedad general.

Por medio de diagnósticos descriptivos, se pretendió iniciar las fases de la metodología Seis Sigma: definir, medir y analizar, que fue planteada para mejorar la calidad de prestación del servicio. Estos análisis, se presentarán a ESE Hospital Lázaro Hernández Lara, para que realicen las intervenciones debidas en pro de mejorar la percepción y la atención de los pacientes, así como preservar su salud que cada día requieren de un buen servicio.

\section{Referencias}

Bigne , M.; Moliner, M. y Sánchez J. (1997). Calidad y Satisfacción en los Servicios Hospitalarios Esencia les y Periféricos. Investigación y Marketing, 57, 55-61.

DNP (2014). Bases del Plan Nacional de Desarrollo 2014-2018. Todos por un nuevo país.

FINDETER, Universidad Industrial de Santander, Universidad del Norte, Microsoft, Fundación Metrópoli (2014). Proyecto Diamante Caribe.

Gutiérrez H. y De la Vara R. (2009). Control Estadístico de Calidad y Seis Sigma. Segunda ed. México: Mc Graw Hill.

Orlandoni G. (2012). Gestión de la Calidad: Control Estadístico y Seis Sigma. Telos: Revista de Estudios Interdisciplinarios en Ciencias Sociales; 14 (2), 269-274.

Ramírez, M.; Pinto de la Sota, S; Serpell, A y Enberg, L. (2007). ¿Seis Sigma en hospitales chilenos? Oikos: Revista de la Escuela de Administración y Economía, 24. 